\title{
Identification and analysis of CEP genes expression in Solanum tuberosum
}

Rutkovskaya E.A.*, Gancheva M.S., Dodueva I.E., Lutova L.A.

Saint-Petersburg State University, St. Petersburg, Russia

*email: rutkovskaya-ea@yandex.ru

Potatoes are one of the most widespread crops in the world. The nitrogen content in the medium affects the development of potato tubers. The development of various parts of the plant is associated with peptides of the CEP group. It is known that in A. thaliana CEPs are responsible for the expression of nitrate transporters depending on the nitrogen concentration in the medium. In $S$. tuberosum, these genes have not been previously discovered. We identified 5 potato genes: StCEP 1, StCEP 3.1, StCEP 3.2, StCEP14, and StCEP15. Homologues of these peptides are AtCEP1, AtCEP3, AtCEP14, and AtCEP 15. Based on the expression data, the following genes were analyzed: StCEP 3.2, StCEP 14 and StCEP15. These genes are highly expressed in potato roots, which may indicate the effect of this peptide on potato tuberization and development of its root system. It is known that CEP peptides, interacting with their receptors (CEPR), activate CEPD peptides, which regulate the expression of nitrate transporters. As a result, we also identified the CEPDL1, CEPDL2, and CEPDL3 genes expressed in potato leaves, as well as receptor genes.

Acknowledgements: This work was supported by a grant RFBR 19-016-00177. 